\title{
Loading Rate Effects on MOE and MOR Distributions in Testing of Small Clear Beech Wood Specimens
}

\author{
Vladislava Mihailović, ${ }^{a, *}$ Mira Miric-Milosavljević, ${ }^{a}$ Marija Djurković, ${ }^{a}$ \\ Goran Mladenović, ${ }^{\mathrm{b}}$ Milos Milosević, ${ }^{\mathrm{b}, \mathrm{c}}$ and Isaak Trajković b,c
}

\begin{abstract}
Distributions of the modulus of elasticity (MOE) and modulus of rupture (MOR) were characterized at three loading rates for small clear beech specimens in static bending. The correlation between MOE and MOR for all three loading rates was significant, but it weakened with increasing load rates. The analysis of the characteristics of empirical distributions, as well as the preliminary selection of the theoretical distributions for MOE and MOR, were performed on the basis of L-moments and L-moment diagrams. According to the standard for testing small specimens, MOE and MOR are determined as the arithmetic mean of the sample. Usage of the arithmetic mean is justified when the analyzed quantity is symmetrically distributed. It was found that the distribution of MOE and MOR is not always symmetric. The loading rate influences the shapes of the MOE and MOR empirical distributions, and consequently the choice of theoretical distribution. The general extreme value distribution stood out as the best one for both MOE and MOR, regardless of the loading rate, and the second overall ranked distribution is the three-parameter Weibull distribution. The loading rate affected the value of the fifth percentile in MOR, when determined from both the empirical and theoretical distributions.
\end{abstract}

DOI: 10.15376/biores.17.1.1818-1835

Keywords: MOE; MOR; Small clear specimens; Beech wood; Loading rate; L-moments; Probability distributions

Contact information: a: Department of Wood Science and Technology, University of Belgrade - Faculty of Forestry, Kneza Višeslava 1, 11030 Belgrade, Serbia; b: University of Belgrade - Faculty of Mechanical Engineering, Kraljice Marije 16, 11120 Belgrade, Serbia; c: Innovation Center of the Faculty of Mechanical Engineering in Belgrade, Kraljice Marije 16, 11120 Belgrade, Serbia;

* Corresponding author: vladislava.mihailovic@sfb.bg.ac.rs

\section{INTRODUCTION}

Wood is an important natural versatile material that can be processed and used for various purposes. The knowledge of the mechanical characteristics of wood is important for the wood product manufacturing industry as well as for industries that use wood products, such as furniture production or construction. The mechanical properties of wood affect its machinability and are therefore important for the wood products industry (Mandić et al. 2015; Porankiewicz et al. 2021). When wood is used as a construction material, its mechanical properties must be known for the purpose of structural design. Two basic properties to assess the stiffness and strength of wood, which are used in the design of wooden structures, are the modulus of elasticity in bending (MOE) and the modulus of rupture in bending (MOR). The MOE and the MOR are determined by testing according to the relevant standards. 
The MOE and MOR of wood are influenced by many factors (e.g. type, origin, growth rate, and age of the tree; the presence of defects, such as knots; moisture content; and density of the wood, etc.). In addition, the mechanical properties of wood depend on the test method. One of the factors that significantly affects the mechanical properties of wood and wood-based materials, which are determined by conventional destructive testing methods, is the loading rate. In general, higher MOE and MOR values are obtained when testing at higher loading rates. In bending tests, the test piece is placed in a testing machine, simply supported, and loaded in bending at one or two points. The loading-head moves downwards at a constant speed. It should be noted that constant loading-head movement actually means a constant rate of deformation. However, in practice it is common to use the term "rate of loading" instead of "rate of deformation" (Gerhards 1977). The term "rate of loading" is also used in this paper, unless the cited authors used another term.

Studies by Liska (1950) and McNatt (1975) were among the first studies on the influence of loading rates on MOE and MOR of wood and wood-based materials. Liska (1950) examined the effect of rapid loading on the compressive and bending strength of solid wood. He examined two softwood species (Sitka spruce and Douglas fir) and two hardwood species (maple and birch). The effect of loading rates on MOE in bending was negligible for all wood species. On the other hand, the bending MOR increased with an increasing loading rate. Deflection at max load in bending for hardwood decreased with an increasing loading rate, but for softwood it was the same regardless of the loading rate. McNatt (1975) showed that the effect of the loading rate on the MOR of particleboard in static bending is very similar to the effect of the loading rate on the MOR of hardboard and solid wood. According to his research, in particleboard the loading rate also affects MOE (MOE increases with an increase in loading rate). Gerhards (1977) gave an extensive review of previous research on the impact of the load duration and loading rate on the strength of wood and wood-based materials. General conclusions are that the impact of deformation rate on bending strength is higher in wet than in dry wood, and that in dry wood the most pronounced effect is the one of the loading rates at tension perpendicular to grain, and then at compression parallel to grain, bending, and shear, which does not differ much for the last three. Compared to solid wood, the loading rate in bending has a slightly greater effect on the strength of hardboard, and a slightly smaller effect on the strength of particleboard. In some more recent studies, Tamrakar and Lopez-Anido (2010) found that for wood plastic composite, both MOE and MOR in bending increase with increasing deformation rate, while MOE was more responsive to variations in deformation rate compared to MOR. Büyüksarı $(2017 \mathrm{a}, \mathrm{b})$ examined the effect of loading rate on some mechanical properties of micro-sized samples of micro-sized oak wood (2017a) and microsized Scots pine wood (2017b). The results for oak wood showed a statistically significant increase in tensile and compression strength, as well as MOE with an increasing loading rate. However, the change in MOR was not statistically significant. On the other hand, the results for Scots pine wood showed that the loading rate had significantly affected compression strength and MOE, but the change in MOR and tensile strength was not statistically significant. For both wood species, it was found that mechanical properties generally decreased with decrease in loading rate.

Due to the influence on the mechanical properties of wood, loading rate is in a way prescribed in the standards for determination MOE and MOR - either explicitly or implicitly (through the given time-to-failure). Bending testing of wood for small clear specimens and structural size specimens are performed in accordance with different standards: the SRPS ISO 13061-3 and -4 (2015) for small clear specimens and the SRPS 
EN 408 (2014) standard for structural size specimens. Testing according to these two standards differs, not only in the sample size and static system, but also in the prescribed procedure. According to ISO 13061-3 (2015), when testing the MOR of small clear specimens, it is prescribed that the failure of a test piece should happen in the time interval of 0.5 to $5 \mathrm{~min}$ from the beginning of loading. According to ISO 13061-4 (2015) the rate of movement of the loading head is not prescribed for determining the MOE in static bending. On the other hand, in the SRPS EN 408 (2014) standard for structural size specimens, the loading rate is defined much more precisely. It is prescribed that when testing MOE in bending, the rate of movement of the loading head shall not be higher than $0.003 \mathrm{~h} \mathrm{~mm} / \mathrm{s}$ (where $\mathrm{h}$ is a height of the test piece cross section). When testing MOR according to this standard, load shall be applied at a constant loading-head movement so adjusted that the maximum load is reached within $(300 \pm 120 \mathrm{~s})$, but this is only for preliminary testing, and target time to reach maximum load for each piece is $300 \mathrm{~s}$.

It is known that the mechanical properties obtained by testing small clear specimens that are considered to be defect-free are better than those determined when testing structural size specimens that contain natural wood anomalies. Nowadays, the characteristic values of the mechanical properties of structural timber are determined on the basis of testing structural size specimens. However, the testing of structural size beams is more expensive and more difficult to carry out: the material itself is expensive due to the quantity, the transport is more difficult and more expensive, more time and space is required for conditioning, and the equipment is more expensive (Krajnc et al. 2019). Therefore, testing on small samples as well as non-destructive methods of testing mechanical properties remains as an alternative to testing on structural size specimens. For example, according to the SRPS EN 384 (2019) standard, alternative methods allowed for determining MOE and MOR are those based on small clear specimen testing for tropical hardwood species (for all hardwood species, in earlier versions of the standard).

Several recent studies have addressed the relationships between MOE and MOR of small clear specimens and structural size specimens (Butler et al. 2016; Krajnc et al. 2019; Nowak et al. 2021). Cunha et al. (2021), went a step further and examined the possibilities for characterizing mechanical properties only through small clear specimens. Namely, according to SRPS EN 384 (2019), the characteristic values of MOE and MOR can be determined by testing small clear specimens only if both small and structural size data are available for at least three similar species. Reduction factors are derived from these data, as the ratio between the mean values of the small clear data and the characteristic values from the structural size data. However, Cunha et al. (2021), stated that the results of some studies show that for the same wood species, but different countries of origin, similar mean values of bending strength can be obtained for small clear specimens, but noticeable differences appear when testing structural size specimens. With this in mind, the previously mentioned authors examined the possibilities of characterizing mechanical properties only by testing small specimens, in order to eliminate the need to test specimens of structural sizes of similar tree species. Based on the tests of Iroco tropical hardwood, they suggested that instead of using the mean value, the characteristic value of bending strength should be determined on the basis of the $5^{\text {th }}$ percentile of small clear specimens, with appropriate reduction factors.

The available literature on the impact of loading rates on MOE and MOR refers only to the mean values, and not to the whole distribution of these mechanical properties of wood. However, if it is of interest to carry out the procedure proposed by the work of Cunha et al. (2021), i.e., if it is necessary to determine the $5^{\text {th }}$ percentile of the small clear 
specimens, then the impact of the loading rate on the whole distribution of MOE and MOR and not only on the mean values should also be examined. Especially because the ISO 13061-3 (2015) and ISO 13061-4 (2015) standards allow a greater "freedom" in the choice of loading rate when examining MOE and MOR than the SRPS EN 408 (2014) standard.

This paper presents the characterization of MOE and MOR distributions for three loading rates, for small clear specimens in static bending according to standards SRPS ISO 13061-4 and SRPS ISO 13061-3. The test was performed on beech (Fagus sylvatica) wood, which is the most widespread and the most important tree species in Serbia. Beech wood is traditionally used for furniture and interior design, but it also has the potential for application in the construction industry, as timber in classic wooden structures and especially for the production of elements for glued laminated structures.

The aims of the investigation of the three loading rates were to: (a) examine possible changes in the character and strength of the relationship between MOE and MOR, (b) describe and compare the characteristics of the MOE and MOR empirical distributions, and (c) perform a probabilistic characterization of the MOE and MOR. The characteristics of the MOE and MOR empirical distributions were examined using L-moments, which are considered to be less error-prone than conventional moments. Further, L-moment diagrams were used for the preliminary selection of theoretical distributions - candidates for the MOE and MOR distributions.

\section{EXPERIMENTAL}

\section{Materials}

The testing was performed on a universal Shimadzu testing machine (Kyoto, Japan) with a maximum capacity of $100 \mathrm{kN}$ (Fig. 1). The test force measurement accuracy is within $\pm 1 \%$ (class 1 of the value indicated for the range $1 / 500$ of the rated capacity). This means that the guaranteed range of the measuring cell is from $200 \mathrm{~N}$ up to $100,000 \mathrm{~N}$ $(100,000 / 500=200)$.

A total of 140 beech wood specimens were cut, with dimensions of $20 \times 20 \times 320$ $\mathrm{mm}$. The samples were conditioned for 30 days, in a room at a temperature of $20 \pm 3{ }^{\circ} \mathrm{C}$ and $65 \pm 5 \%$ absolute air humidity. During specimen preparation, care was taken to have an equal number of specimens in each group with the same wood fiber orientation and the same growth rings. Information on the origin, size, or age of the tree from which the board was cut could not be traced.

\section{Methods}

The testing was performed in accordance with standards SRPS ISO 13061-3 (2015) and SRPS ISO 13061-4 (2015). A concentrated force acted in the middle of a specimen (Fig. 1). This is the so-called three-point bending test. The distance between the supports was $280 \mathrm{~mm}$. The radii of the loading head and the supports were $15 \mathrm{~mm}$. The speed of movement of the loading head was constant. The MOE and MOR testing was performed on the same specimens.

Three loading rates, i.e., speeds of loading head movement, were selected on the basis of a trial measurement. The criterion for selecting the loading rate was the measured time-to-failure of the test specimens. The loading rate of $1 \mathrm{~mm} / \mathrm{min}(0.016 \mathrm{~mm} / \mathrm{s})$ was chosen so that the time-to-failure was close to the upper limit of the prescribed time range of 0.5 to $5 \mathrm{~min}$. The second selected loading rate of $3 \mathrm{~mm} / \mathrm{min}(0.05 \mathrm{~mm} / \mathrm{s})$ led to sample 
failure in the prescribed range of time-to-failure. The third selected loading rate of 10 $\mathrm{mm} / \mathrm{min}(0.166 \mathrm{~mm} / \mathrm{s})$ caused specimen failure close to the lower limit of the prescribed time-to-failure range.

Mean density was measured in accordance with the SRPS ISO 13061-2 (2015) standard and equilibrium moisture content according to standard SRPS ISO 13061-1 (2015).

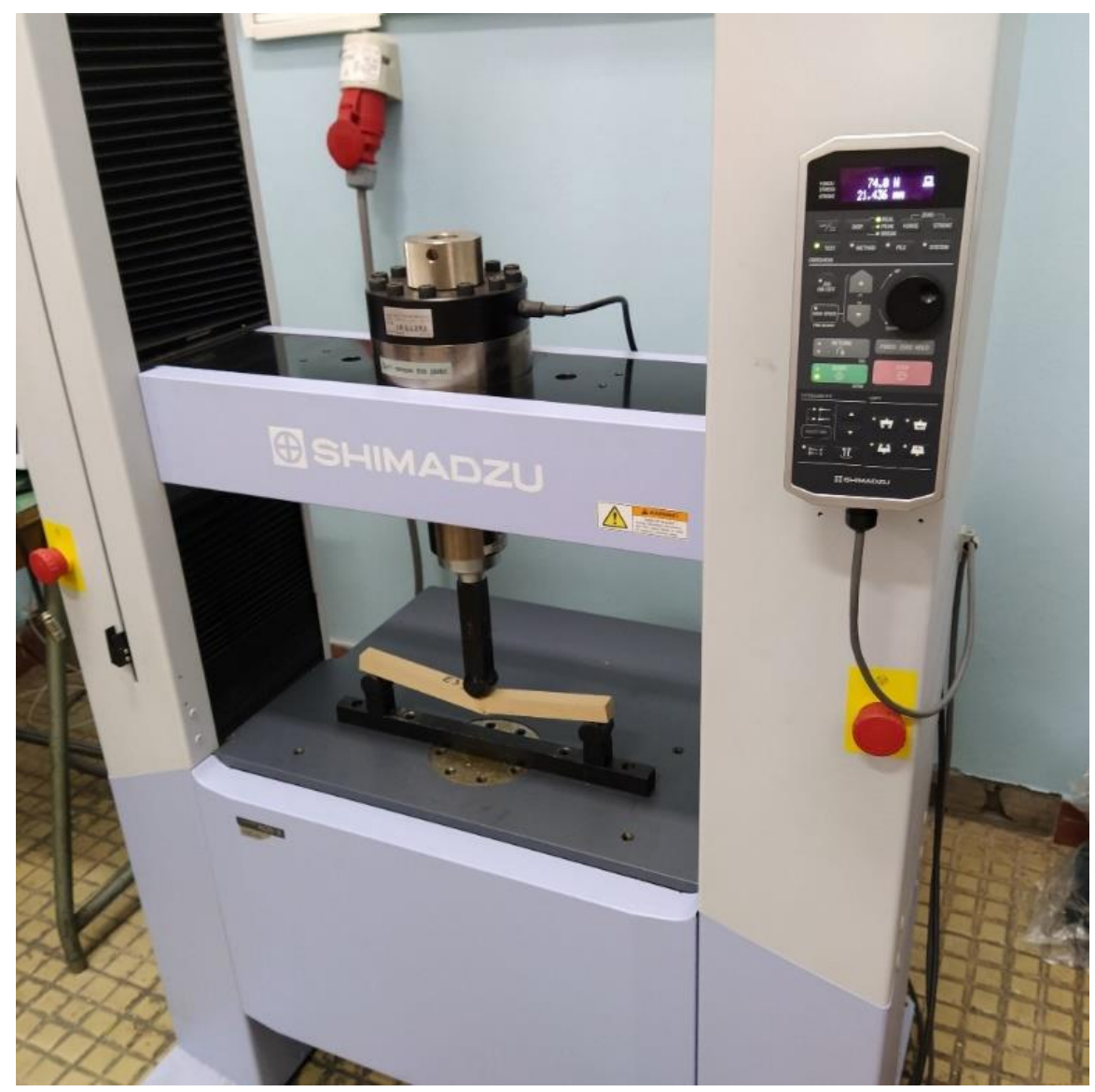

Fig. 1. Three point bending test setup and testing equipment

\section{Statistical Analysis}

The MOE and MOR series for three different loading rates were subjected to statistical analysis in several steps. First, the correlation between MOE and MOR was examined. Second, the change in the characteristics of empirical MOE and MOR distributions was evaluated, and a preliminary selection of theoretical distributions candidates for the MOE and MOR models - was performed. Third, the agreement between the selected models of theoretical distributions and empirical MOE and MOR distributions was assessed.

The properties of a distribution are described by moments and dimensionless parameters-moment ratios. For example, the coefficient of variation $C_{v}$ is used as a measure of sample variability, the coefficient of skewness $C_{s}$ as a measure of asymmetry, and the coefficient of kurtosis $C_{k}$ describes the properties of the distribution "tails". Analogous to these "conventional" parameters, both L-moments and L-moment ratios are used to describe distribution properties. The first two L-moments and L-moment ratios, as well as 
L-skewness and L-kurtosis are counterparts to conventional moments and product moment ratios and can be understood as measures of location, scale, skewness, and kurtosis (Hosking 1990). The advantages of L-moments over conventional moments are often highlighted in the literature (e.g., Hosking 1990; Vogel and Fennessey 1993; Sankarasubramanian and Srinavasan 1999), and because of this L-moments have long had a major application in hydrology and climatology. Since they are calculated as linear combinations of an ordered sample, the estimates of L-moments are subject to smaller errors than conventional moments, which are calculated by the exponentiation of differences between the series members and the mean value of the series. Therefore, Lmoments can be expected to show less sensitivity to variations in the sample or errors in measurements (extremes are far from the mean value).

Changes in the characteristics of the MOE and MOR empirical distributions with changes in the loading rate were analyzed on the basis of product moment ratios and Lmoment ratios. Table 1 shows product moment ratios and L-moment ratios in parallel, with their names and notation used in this paper.

Moment ratio diagrams (MRDs) are often used to identify the appropriate theoretical distribution (or several candidates) as a possible model for the distribution of the observed quantity. Following the example of MRDs which show product moment ratios, Hosking (1990) introduced L-moment ratio diagrams. L-moment ratio diagrams show the relationship between $L C_{v}$ and $L C_{s}$ for two-parameter, and $L C_{k}$ and $L C_{s}$ for threeparameter distributions, respectively. Explicit expressions $L C_{v}=f\left(L C_{s}\right)$ and $L C_{k}=f\left(L C_{s}\right)$ for diagram construction can be found in the literature (e.g., Vogel and Wilson 1996; Rao and Hamed 2000). In this paper, the preliminary selection of theoretical distributions candidates for the MOE and MOR theoretical models was performed on the basis of Lmoment diagrams.

Table 1. Product Moment Ratios and L-Moment Ratios with Names and Notation Used in this Paper

\begin{tabular}{|l|c|c|c|c|c|c|}
\hline & \multicolumn{3}{|c|}{ Product Moment Ratios } & \multicolumn{3}{c|}{ L-moment Ratios } \\
\hline \multirow{2}{*}{ Name } & \multicolumn{3}{|c|}{ Coefficient of } & \multicolumn{3}{c|}{ L-coefficient of } \\
\cline { 2 - 7 } & Variation & Skewness & Kurtosis & Variation & Skewness & Kurtosis \\
\hline Notation & $C_{V}$ & $C_{S}$ & $C_{k}$ & $L C_{V}$ & $L C_{S}$ & $L C_{k}$ \\
\hline
\end{tabular}

Finally, common goodness-of-fit tests were applied to assess the agreement between the empirical distributions of MOE and MOR and the selected theoretical distributions. The fit of selected distributions was compared based on the results from the Cramer von Mieses and Kolmogorov-Smirnov goodness-of-fit tests (D'Agostino and Stephens 1986), as well as the root mean square error (RMSE) between the empirical and theoretical distributions.

\section{RESULTS AND DISCUSSION}

The Grubs-Beck test (at significance level $\alpha=10 \%$ ) was used to detect outliers. According to this test, one lower outlier was identified for the MOR series, at the loading rate of $10 \mathrm{~mm} / \mathrm{min}$. The specimen for which this result was obtained was excluded from further analysis. The upper outliers were not detected.

Mihailovic et al. (2022). "Loading rate \& MOE \& MOR," BioResources 17(1), 1818-1835. 1823 


\section{Measurement Results and the Basic Statistical Indicators of Measured Quantities}

Table 2 shows the measurement results of three groups of specimens loaded at different loading rates. The table shows the number of samples for each group, as well as the basic data for density, fracture force, maximum deflection, and time-to-failure. The average values of samples measured moisture content according to SRPS ISO 13061-1 (2015), which was 7.36\%.

One-way analysis of variance (ANOVA) test (for the significance level $\alpha=0.05$ ) revealed that the difference between the mean values of density of the three specimen groups was not statistically significant $(F(2,127)=1.12, p=0.329)$. Further, the applied Levene's test for the equality of variance indicated that the difference in the variance of the density of the three specimen groups was not statistically significant $(F(2,127)=1.29, p$ $=0.280$ ).

As expected, the loading rate greatly influenced the time-to-failure (Table 2). As the loading rate increased, the time-to-failure and the range of time in which failure occurred noticeably decreased. A number of samples from the group loaded at a rate of 1 $\mathrm{mm} / \mathrm{min}$ experienced a failure after more than $5 \mathrm{~min}$.

Table 2. Basic Statistical Indicators for Density, Fracture Force, Deflection, and Time-to-Failure of Three Groups of Specimens Loaded at Selected Loading Rates

\begin{tabular}{|c|c|c|c|c|c|c|c|c|c|c|c|}
\hline \multirow{2}{*}{$\begin{array}{c}\text { Loading } \\
\begin{array}{c}\text { Rate } \\
(\mathrm{mm} / \mathrm{min})\end{array}\end{array}$} & $\begin{array}{c}\text { No. } \\
\text { of } \\
\text { Spec. }\end{array}$ & \multicolumn{2}{|c|}{$\begin{array}{c}\text { Density } \\
\left(\mathrm{kg} / \mathrm{m}^{3}\right)\end{array}$} & \multicolumn{2}{c|}{$\begin{array}{c}\text { Fracture } \\
\text { Force }(\mathrm{N})\end{array}$} & \multicolumn{2}{c|}{$\begin{array}{c}\text { Deflection } \\
(\mathrm{mm})\end{array}$} & \multicolumn{4}{c|}{$\begin{array}{c}\text { Time-to-failure } \\
(\mathrm{s})\end{array}$} \\
\cline { 3 - 14 } & $\begin{array}{c}\text { Mean } \\
\text { dev. }\end{array}$ & Mean & $\begin{array}{c}\text { St. } \\
\text { dev. }\end{array}$ & Mean & $\begin{array}{c}\text { St. } \\
\text { dev. }\end{array}$ & Min. & Max. & Mean & $\begin{array}{c}\text { St. } \\
\text { dev. }\end{array}$ \\
\hline 1 & 44 & 700 & 62.8 & 2295 & 318.4 & 10.01 & 1.17 & 442 & 730 & 604 & 70.3 \\
\hline 3 & 43 & 707 & 60.7 & 2372 & 347.3 & 10.13 & 1.29 & 131 & 250 & 203 & 25.8 \\
\hline 10 & 43 & 719 & 51.5 & 2450 & 303.8 & 10.33 & 1.34 & 40 & 75 & 62 & 8.1 \\
\hline
\end{tabular}

\section{Results for MOE and MOR}

The basic statistical indicators of the MOE and MOR series are shown in Table 3. Hereinafter, the series of the MOE obtained at loading rates of 1,3 and $10 \mathrm{~mm} / \mathrm{min}$ will have designations of MOE1, MOE3, and MOE10. Analogously, the MOR series will be designated MOR1, MOR3, and MOR10. Along with the mean values of MOE and MOR, Table 3 gives the percentage differences between the given and the reference value in parentheses (the mean value of the MOE1 and MOR1 series was taken as the reference value). The MOE3 series had the lowest mean value, while the highest value was recorded for MOE10, but the differences between the mean values of all three series were very small. The mean value of MOR increased with an increasing loading rate. The difference between MOR3 and MOR1 was small and amounted to $2.5 \%$, and the difference between MOR10 and MOR1 was much bigger, reaching 6.8\%. The MOE and MOR series for different loading rates were also compared using the one-way ANOVA test. For the significance level $\alpha=0.05$, it was shown that the differences between the mean values of these series were not statistically significant, either for $\operatorname{MOE}(F(2,127)=0.84, p=0.436)$ or for MOR $(F(2,127)=2.57, p=0.080)$. If considering only the mean value, changes in the loading rate had a higher impact on MOR than on MOE. 
Table 3. Basic Descriptive Statistics for MOE and MOR in the Bending Test for Three Loading Rates

\begin{tabular}{|c|c|c|c|c|c|c|}
\hline & \multicolumn{3}{|c|}{ MOE (MPa) } & \multicolumn{3}{|c|}{ MOR (MPa) } \\
\hline $\begin{array}{l}\text { Loading } \\
\text { Rate }\end{array}$ & $1 \mathrm{~mm} / \mathrm{min}$ & $3 \mathrm{~mm} / \mathrm{min}$ & $10 \mathrm{~mm} / \mathrm{min}$ & $1 \mathrm{~mm} / \mathrm{min}$ & $3 \mathrm{~mm} / \mathrm{min}$ & $10 \mathrm{~mm} / \mathrm{min}$ \\
\hline Mean* & 12926 & $\begin{array}{c}12886 \\
(-0.3 \%) \\
\end{array}$ & $\begin{array}{c}13328 \\
(+3.1 \%) \\
\end{array}$ & 122.3 & $\begin{array}{c}125.4 \\
(2.5 \%) \\
\end{array}$ & $\begin{array}{c}130.6 \\
(6.8 \%) \\
\end{array}$ \\
\hline St. dev. & 1650 & 1753 & 1862 & 17.1 & 18.1 & 16.1 \\
\hline$C v$ & 0.127 & 0.136 & 0.123 & 0.140 & 0.144 & 0.123 \\
\hline Cs & -0.100 & -0.263 & -0.010 & -0.038 & -0.164 & -0.475 \\
\hline$C k$ & 2.131 & 2.252 & 1.564 & 2.131 & 1.850 & 1.994 \\
\hline$L C v$ & 0.073 & 0.081 & 0.081 & 0.081 & 0.084 & 0.070 \\
\hline LCs & -0.016 & -0.009 & -0.003 & -0.009 & -0.047 & -0.137 \\
\hline LCK & 0.082 & 0.062 & -0.056 & 0.062 & -0.001 & -0.005 \\
\hline
\end{tabular}

Figure 2 shows the correlation between time-to-failure and MOE (Fig. 2a) and timeto-failure and MOR (Fig. 2b), which clearly shows the difference in the time range in which the failure occurred for these three groups of samples loaded at different loading rates. The same figure shows points representing mean time-to-failure $v s$. mean MOE (MOR) for all three groups of samples.
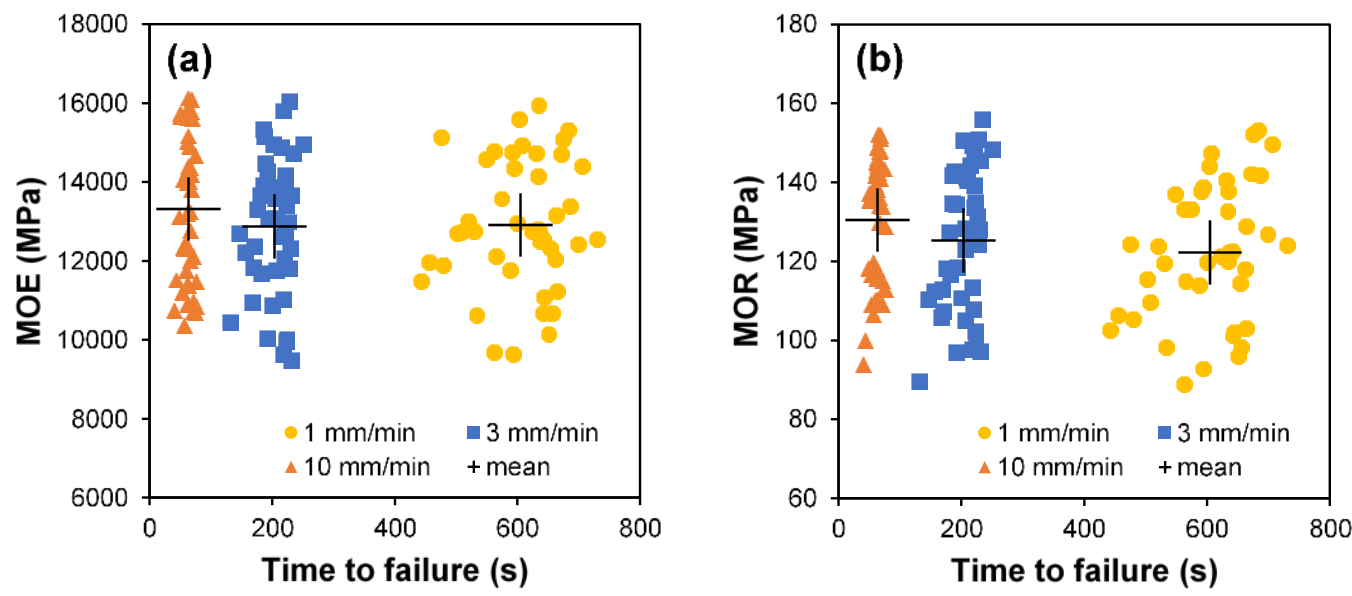

Fig. 2. Time-to-failure vs. MOE (a) and time-to-failure vs. MOR (b), for the three loading rates

Regression analysis clearly indicated a strong correlation between MOE and MOR for all three loading rates. However, the coefficient of determination decreased with an increasing loading rate, i.e., the correlation between MOE and MOR weakened. The coefficients of determination $\left(\mathrm{R}^{2}\right)$ for the regression lines for loading rates $1 \mathrm{~mm} / \mathrm{min}, 3$ $\mathrm{mm} / \mathrm{min}$, and $10 \mathrm{~mm} / \mathrm{min}$ were $0.862,0.842$ and 0.767 , respectively. Figure 3 shows the linear regression plot between MOR and MOE (MOE is an independent variable), the equation of the linear regression model, as well as the limits for the $95 \%$ confidence (mean) interval and $95 \%$ prediction interval, for all three loading rates. 

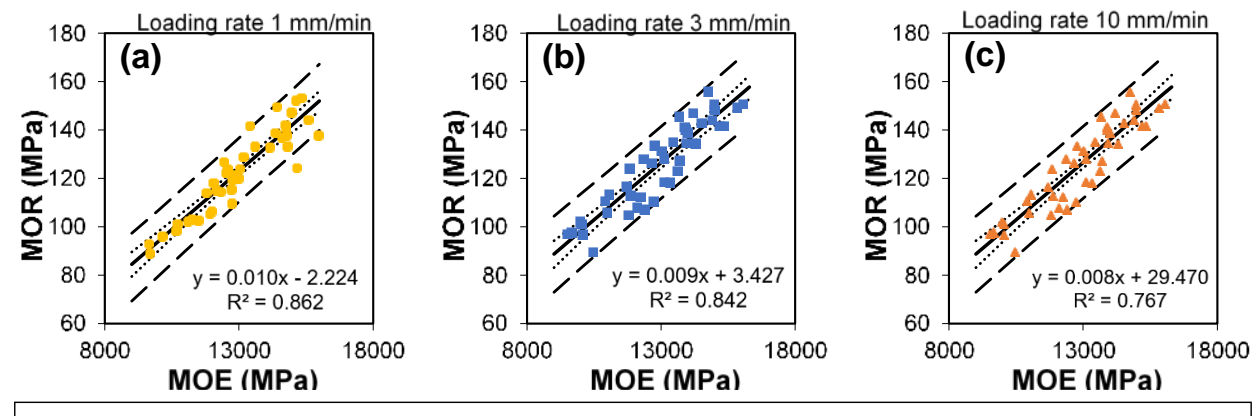

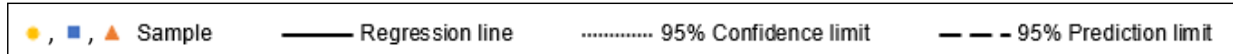

Fig. 3. Relationship between MOE and MOR for different loading rates

\section{L-Moment Ratio Diagrams}

It was shown in results for MOE and MOR that the influence of the loading rate on the mean values of MOE and MOR was not statistically significant. Still, monitoring of only the mean value does not provide much information about the characteristics of the empirical distributions of MOE and MOR, and whether these characteristics change with the change in the loading rate. Therefore, other statistical indicators, higher-order moments and L-moments (shown in Table 3), which describe the shape of distribution, were included in the analysis of the MOE and MOR series.

Figures 4 and 5 show diagrams of L-moments that were used for the comparison of sample L-moment ratios for MOE and MOR with the population values of commonly used two- and three-parameter frequency distributions. Abbreviations for the names of the distributions in the diagrams and in the paper are: $\mathrm{N}$ - Normal distribution; GPA2 and GPA, two and three-parameter Pareto; LN2 and LN3 - two and three-parameter Lognormal; GAM2 - two-parameter Gamma; W2 and W3 - two and three-parameter Weibull; GLOG - General logistic; GEV - Generalized extreme value; and P3 - Pearson type 3 distribution. The diagrams cover only the area in which sample L-moment ratios of MOE and MOR series are located. Figures $4 \mathrm{a}$ and 5a show L-moment diagrams for the two-parameter distributions, $L C_{s} v s$. $L C_{v}$. The diagrams of $L C_{s} v s$. $L C_{k}$ for three-parameter distributions are shown in the Figs. $4 \mathrm{~b}$ and $5 \mathrm{~b}$. The diagrams in Figs. $4 \mathrm{c}$ and $5 \mathrm{c}$ serve to compare the positions of empirical points $\left(L C_{s}, L C_{k}\right)$ for logarithms of MOE and MOR with the theoretical curve for P3 distribution, used as an indicator of agreement of the LP3 distribution with the MOE and MOR series. Namely, for the LP3 distribution there are no explicit expressions for the relationship between $L C_{s}$ and $L C_{k}$, so a simple method is applied here by which points $\left(L C_{s}, L C_{k}\right)$ are plotted on the diagram for a logarithmically transformed sample and their position is compared to the theoretical curve for the P3 distribution.

It can be seen in the $L C_{s}-L C_{v}$ diagrams for MOE and MOR (Figs. 4a and 5a) that the L-coefficient of variation for both MOE and MOR was almost constant. On the other hand, $L C_{s}$ changed with a changing loading rate, and all series had a negative $L C_{s}$ (as well as $C_{s}$, Table 3), i.e., they were left-skewed. For MOE the lowest $L C_{s}$ was recorded for MOE3, and the MOE1 and MOE10 series had very little negative skewness. LCs decreased with an increasing loading rate for MOR, and it became more negative. The MOE10 was practically a symmetric series, as was MOR 1 . The value of the asymmetry coefficient close to zero meant that the application of the normal distribution should be considered (for the $\mathrm{N}$ distribution, $L C_{s}=0$ and $L C_{k}=0.1226$ ). All points for MOE and MOR, except MOR10, 
fell into the space between the curves of the W2 and LN2 distributions. Given that all series had a negative asymmetry, the GAM2 and LN2 distributions were not considered here.
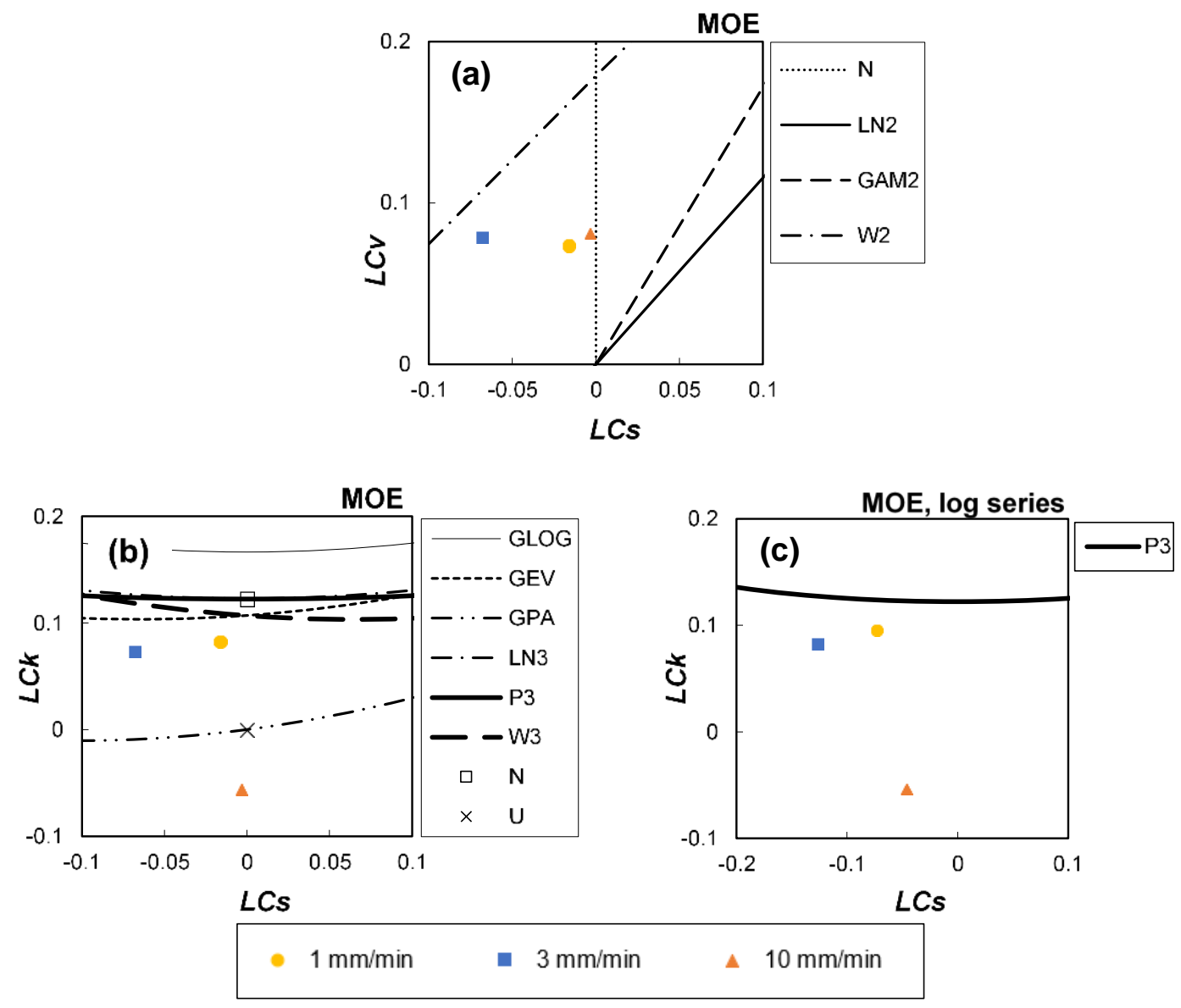

Fig. 4. L-moment diagrams for $\mathrm{MOE}$ : The $L C_{s} v s$. $L C_{V}$ diagram for two-parameter distributions (a), $L C_{s}$ vs. $L C_{k}$ for three-parameter distributions (b), and $L C_{s} v s$. $L C_{k}$ for log sample (c)

The $L C_{s} v s . L C_{k}$ diagrams (Figs. $4 \mathrm{~b}$ and $5 \mathrm{~b}$ ), show the relationship between the two indicators of distribution shape. The first thing that catches the eye is the low $L C_{k}$ compared to most theoretical distributions. It is obvious that empirical distributions belong to the socalled "short-tailed" distributions. For all three loading rates the $L C_{k}$ of MOR and MOE was lower than for the normal distribution, but the empirical points also lay below the theoretical lines for all three-parameter distributions except GPA. For both MOE and MOR, $L C_{k}$ decreased with an increasing loading rate, which was more pronounced for MOE. The series MOR1 had the highest $L C_{k}$ among the MOR series, and there was almost no difference between series MOR3 and MOR10. Series MOE1 and MOR1, and then MOE3, had the highest $L C_{k}$, i.e., the closest one to the lines for the group of theoretical distributions LN3, P3, GEV, and W3. In that group of curves for several theoretical distributions located above the points for MOE and MOR, the lowest curve was for GEV and then for W3. The conclusion is that according to this diagram, the GLOG distribution should be excluded from consideration, and for other distributions it should be seen how it will fit to the sample. 

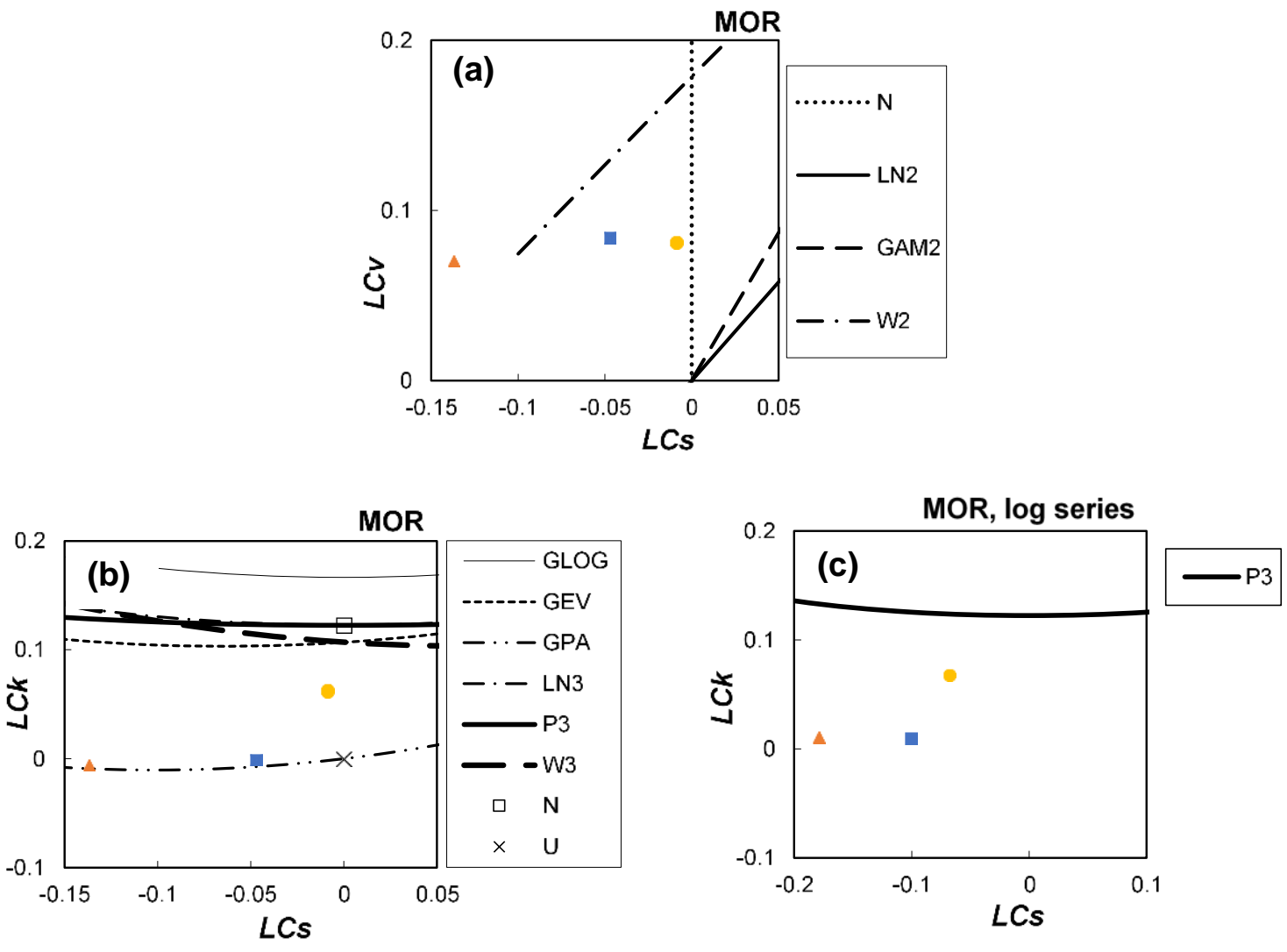

$$
1 \mathrm{~mm} / \mathrm{min} \quad 3 \mathrm{~mm} / \mathrm{min} \quad \Delta 10 \mathrm{~mm} / \mathrm{min}
$$

Fig. 5. L-moment diagrams for MOR: The $L C_{s} v s$. $L C_{v}$ diagram for two-parameter distributions (a), $L C_{s}$ vs. $L C_{k}$ for three-parameter distributions (b), and $L C_{s} v s$. $L C_{k}$ for log sample (c)

Figures $4 \mathrm{c}$ and $5 \mathrm{c}$ show diagrams for the logarithms of MOE and MOR, with a curve for the P3 distribution. The already mentioned problem with a low $L C_{k}$ can also be seen here. Secondly, it is known that logarithmic transformation reduces the coefficient of skewness, so that the logarithmic MOE and MOR series became more left-skewed.

The normal distribution is most often assumed for the MOE distribution, and a normal, log-normal, and Weibull distribution for the MOR distribution (Gupta et al. 1992; Owens et al. 2018). The application of the Weibull's model for strength properties is based on the theoretical "weakest link" concept. This concept was adapted for application to wood by Bohannan (1966) who established the size to strength relationship, which makes it possible to transpose the bending strength results obtained on small samples to structural size beams. These relations are still used today in several American standards (Evans et al. 2019). However, in a recent study, Owens et al. (2018) presented the results of a research of MOE and MOR distribution testing for "a full lumber population of southern pine 2 " $\times$ 4 ", produced in a single mill on a single day" subjected to the static bending test. These authors fit several distributions to the MOR and MOR data and found that neither MOE nor MOR was well-fit by a Weibull distribution. According to the authors' results mill-run MOE might be adequately modeled by a normal distribution or a mixture of two normal distributions, and mill-run MOR might be adequately modeled by a skew normal distribution or a mixture of two normals. 
The fact that the MOE and MOR series were left-skewed limited the selection of distributions for MOE and MOR (although the absolute values of LCs (or Cs) were very low for some series). Yet, it seems that the crucial indicator here was a low kurtosis of empirical distributions. In view of all the above, the following distributions were selected for further consideration: N, LN3, W2, W3, GEV, P3, LP3, as well as GPA, and they were the same for MOE and MOR. The following methods were applied for the estimation of distribution parameters: the method of moments for N, P3, and LP3, maximum likelihood estimation for W2 and W3, the method of moments combined with quantile lower bound estimator (Stedinger 1980) for LN3, and the method of L-moments for GEV and GPA.

\section{Distribution Fitting}

The selected three-parameter distributions can have an upper and/or lower limit. By checking the position of the lower and/or upper limit of the distribution in relation to the minimum and maximum values of the MOE and MOR series, it was determined that the GPA distribution must be excluded from further consideration. This distribution was limited in both cases from both the bottom and the top, and in all series except for MOE10, the upper limit was below the recorded highest data values.

The fit of distributions was comparatively assessed based on RMSE and the results of Cramer von Mieses (C-M) and Kolmogorov-Smirnov (K-S) goodness of fit tests. (It turned out that all considered distributions passed the criteria set in these two tests, for the significance level $\alpha=0.05$ ). The aim was not to formally select the "best" distribution, but to compare the ability of the selected theoretical distributions to fit to MOE and MOR series for different loading rates.

The relative values of test statistics were calculated to facilitate the comparison of theoretical models. In all three tests, a lower value of test statistics meant better fit of the theoretical model to the empirical distribution, i.e., the best-fit distribution for each test would be the one with the lowest value of test statistics. The relative value of test statistics of the $i$-th distribution $\left(T S_{i, r e l}\right)$ was calculated for each test as the ratio of the lowest value of test statistics (minTS) and test statistics for a given distribution $\left(T S_{i}\right)$ :

$T S_{i}$, rel $=\min T S / T S_{i}$

The $T S_{i, r e l}=1$ would be obtained for the distribution with the best result, and for all others it would be lower than 1. Figure $6(a, b, c)$ shows a comparison of selected theoretical distributions for MOE, and Fig. 6 (d, e, f) for MOR at all three loading rates.

According to the test results, GEV performed best for both MOE and MOR, for all three loading rates. The second-best ranked distribution was W3. This distribution performed slightly worse only for MOE1 and MOR10. Such consistent results were not obtained for the other considered theoretical models, neither for MOE nor for MOR. The normal distribution achieved the best result for MOE1, and it proved to be good for the other two series with low asymmetry - MOE10 and MOR1. The fit of two-parameter Weibull distribution to the sample data was comparable to the fit of three-parameter Weibull distribution only for MOR10. The test results were in agreement with the statements about the properties of the empirical series that were made based on the analysis of L-moment diagrams. Theoretical curves for the best ranked distributions, GEV and W3, were the lowest in the $L C_{s} v s . L C_{k}$ diagrams, i.e., these distributions had the lowest $\mathrm{LC}_{\mathrm{k}}$ (Figs. $4 \mathrm{~b}$ and $5 \mathrm{~b}$ ). For those series that had a low asymmetry coefficient, the normal distribution also showed good results, and for the series MOE1 and MOR1, LC $k$ was the closest to the one for the normal distribution. 


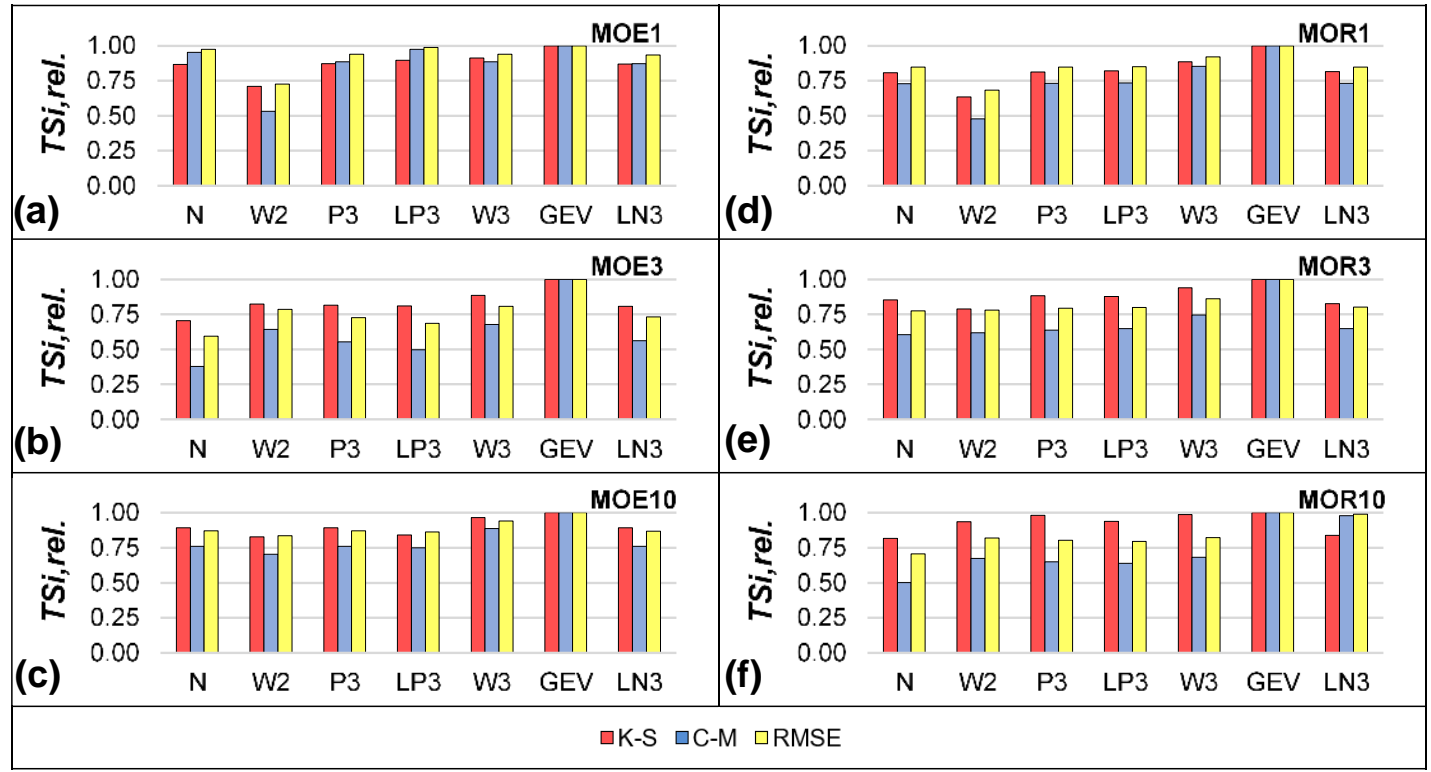

Fig. 6. Relative values of test statistics, $T S_{i}$, rel, for $\operatorname{MOE}(a, b, c)$ and for MOR (d, e, f)

According to the L-moment diagrams, it is predicted that the basic problem in modeling MOE and MOR will be that the $\mathrm{LC}_{\mathrm{k}}$ of empirical distributions is lower than the LCk of the theoretical ones, i.e., the fact that empirical distributions have the so-called "shorter tails" and that they are underdispersed compared to all considered theoretical distributions. Probability plots (Figs. 7 and 8) show the changes in the properties of the empirical distributions of MOE and MOR with a change in the loading rate, as well as the ability of selected theoretical models to adapt to those changes.
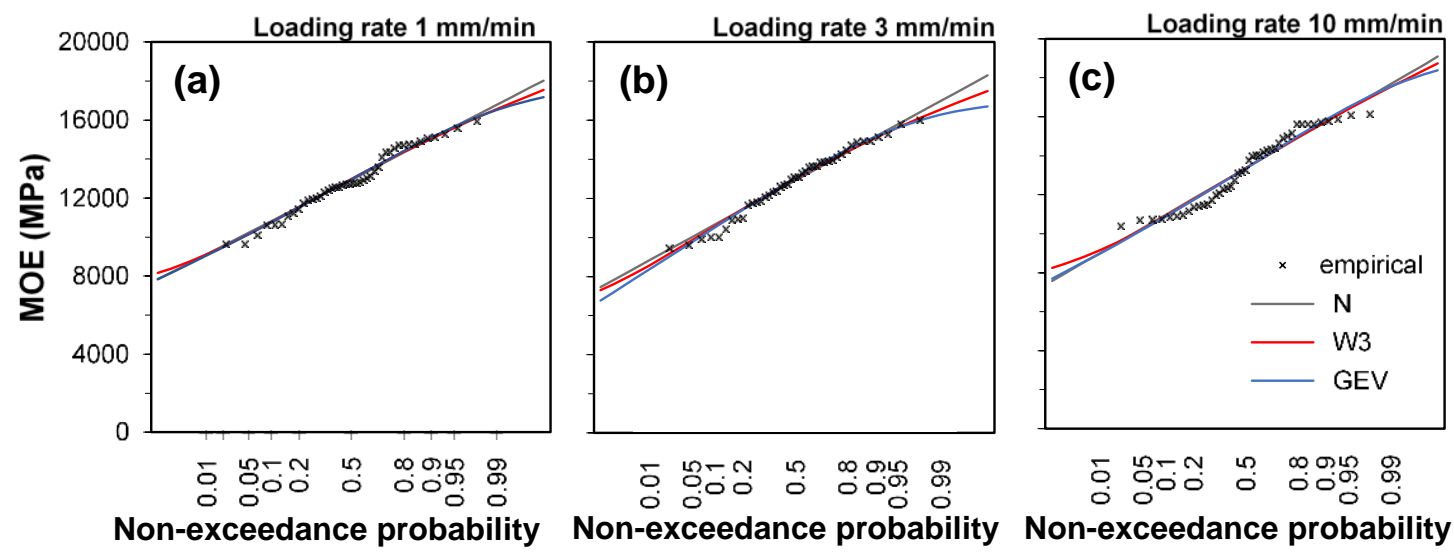

Fig. 7. Probability plots for empirical and candidate theoretical distributions for three loading rates - MOE

The probability plots clearly show that the shape of the empirical distribution very much depends on the loading rate (normal probability paper was used due to the comparison of several models). Figure 7 shows probability diagrams for MOE, for three loading rates. The empirical series and three fitted theoretical distributions, GEV, W3, and $\mathrm{N}$, are shown for each loading rate. Due to the low values of $L C k$ and $C k$ the empirical distributions for MOE had the shape of a slanted letter " $\mathrm{S}$ ", and with an increasing loading 
rate it became more pronounced (i.e., distributions have shorter "tails"). As far as MOR was concerned, (Fig. 8) MOR3 had a more pronounced "S" shape, and the problem with the MOR10 series was that it had two fairly clearly separated groups of data. The highest loading rate $(10 \mathrm{~mm} / \mathrm{min})$ produced distorted empirical MOE and MOR series, and this is a fact that needs further attention.
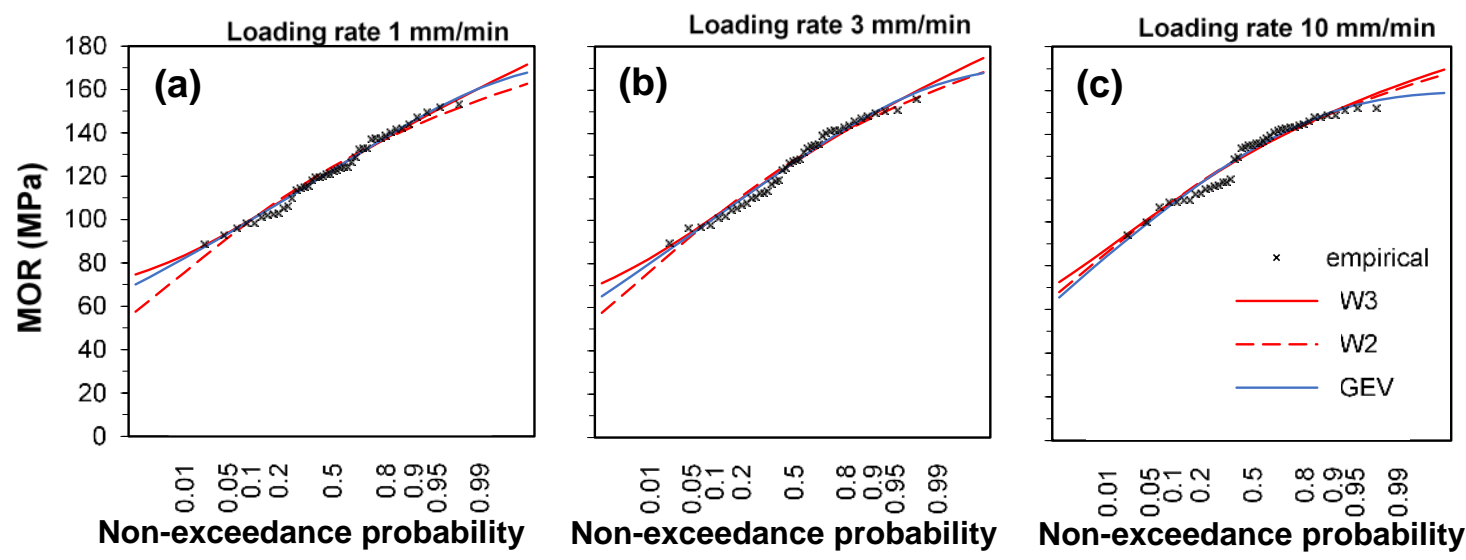

Fig. 8. Probability plots for empirical and candidate theoretical distributions for three loading rates - MOR

The fit of the theoretical models to the lower tail of empirical distribution for MOR was estimated on the basis of the lower $5^{\text {th }}$ percentile values (MOR 0.05 ). These values for empirical distributions were calculated based on the methodology from standard SRPS EN 384 (2019), and for the theoretical ones using the inverse distribution function.

Figure 9a shows relative differences (RD) $(\%)$, between the theoretical values of the fifth percentile of the GEV and $\mathrm{W} 3$ distributions in relation to the empirical $5^{\text {th }}$ percentile (as the reference value), for three loading rates, denoted as MOR $1_{0.05}$, MOR $3_{0.05}$, and MOR10 0.05 . In general, these differences were not large. When comparing the MOR $\mathrm{M}_{0.05}$ values evaluated from empirical or theoretical models, for the loading rates of 1 and 10 $\mathrm{mm} / \mathrm{min}$, there was small difference, and for the loading rates of $3 \mathrm{~mm} / \mathrm{min}$ the theoretical models gave slightly lower values than the empirical ones. There was almost no difference between the values of MOR $1_{0.05}$, and for MOR $10_{0.05}$ the differences were slightly higher, but still below $1 \%$ in terms of absolute value. The biggest difference in absolute value between the theoretical and empirical MORs 0.05 was obtained for the loading rate of 3 $\mathrm{mm} / \mathrm{min}$, but it was also small and amounted to $3.6 \%$ (for GEV) and $2.6 \%$ (for W3).

The change of the fifth percentile with a changing loading rate, for the empirical, GEV and W3 distributions, is shown in Fig. 9b. The MOR $1_{0.05}$ values were taken as reference values. It can be seen here that the change in the loading rate can noticeably affect the change in the fifth percentile, both in the empirical and theoretical distributions. The value of MOR 0.05 increased with an increasing loading rate, and the highest RD was between MOR $10_{0.05}$ in relation to MOR $1_{0.05}$. It reached $8.1 \%$ for the empirical distribution, $7.2 \%$ for the GEV distribution, and $8.3 \%$ for the W3 distribution. Values for RD between the $5^{\text {th }}$ percentile at different loading rates were noticeably higher than the RD between the empirical and theoretical $5^{\text {th }}$ percentile values for the same loading rate. 

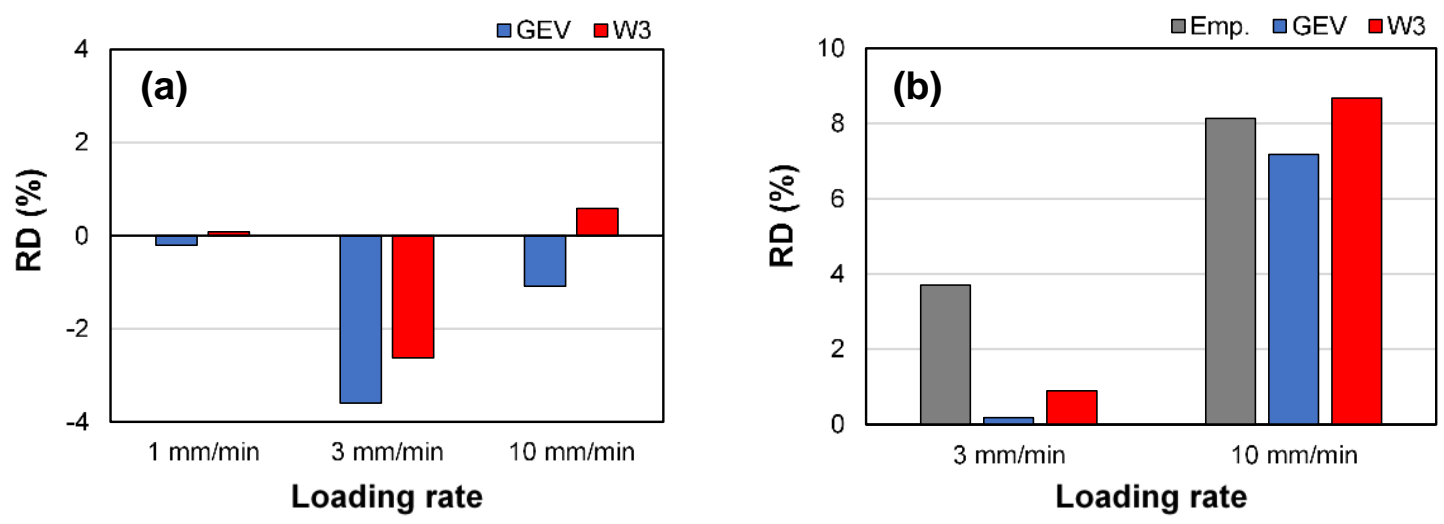

Fig. 9. (a) RD (\%), between the theoretical MOR 0.05 distributions of GEV and $W 3$ and the empirical $\mathrm{MOR}_{0.05}$, for three loading rates; (b) RD (\%), between the MOR $\mathrm{R}_{0.05}$ obtained at the loading rates of $3 \mathrm{~mm} / \mathrm{min}$ and $10 \mathrm{~mm} / \mathrm{min}$ in relation to the $\mathrm{MOR}_{0.05}$ at a loading rate of $1 \mathrm{~mm} / \mathrm{min}$, for the empirical, GEV, and W3 distributions

According to the standard for testing small samples, MOE and MOR should be determined as the arithmetic mean of the sample. Also, the available literature on the impact of loading rates on MOE and MOR refers only to the mean values. Indeed, the change in the mean values of the MOE and MOR series when changing the loading rate, shown in the Table 3, was small, and ANOVA test indicated that those changes were not statistically significant. The use of the arithmetic mean is justified when the analyzed quantity is symmetrically (e.g. normally) distributed. However, it was shown here that distribution of MOE and MOR was not always symmetrical, since skewness varied with change in loading rate. In general, the loading rate influenced the MOE and MOR empirical distribution shape (expressed through the indicators of skewness and kurtosis, Figs. 4 and 5) and, consequently, the choice of theoretical distribution (Figs. 7 and 8).

The theoretical distribution for MOE and MOR has to be flexible in terms of shape. Therefore, the two three-parameter distributions were singled out according to the applied tests and probability plot. The GEV distribution proved to be the best and least sensitive to changes in the loading rate of all the distributions considered in this analysis. Moreover, the GEV model has not been applied for MOE and MOR assessment so far, according to the available literature. The distributions often used for MOE and MOR in the literature (Gupta et al. 1992; Owens et al. 2018), W3 proved to be the best. Normal and W2 distributions were difficult to adapt to changes in the shape of empirical distribution.

\section{CONCLUSIONS}

1. A significant correlation was found between modulus of elasticity (MOE) and modulus of rupture (MOR) for all three loading rates, but this correlation weakened with an increasing loading rate.

2. The mean value of MOR was more sensitive to changes in the loading rate than the mean value of MOE. With an increase in the loading rate from $1 \mathrm{~mm} / \mathrm{min}$ to 10 $\mathrm{mm} / \mathrm{min}$, the mean MOE increased by $3.1 \%$ and the mean MOR by $6.8 \%$. However, it was shown that the differences between the mean values of these series were not statistically significant, neither for MOE nor for MOR. 
3. In general, it was concluded that L-moments and L-moment diagrams were a useful tool in describing and analyzing the characteristics of empirical distributions, and in the distribution selection as well. The shape of the empirical MOE and MOR distributions, expressed through indicators of skewness and kurtosis, depended on the loading rate. The coefficient of variation did not change noticeably with a change in the loading rate. The empirical MOE and MOR distributions, for all three loading rates belong to short tails distributions.

4. The variability of indicators of the shape of empirical distributions when changing the loading rate influenced the acceptability of theoretical models. Two three-parameter distributions belonging to the family of extreme value distributions were singled out. The general extreme value distribution stood out as the most versatile and consistently best for both MOE and MOR, regardless of the loading rate. The second overall ranked distribution was the three-parameter Weibull distribution. Other three-parameter distributions, as well as two-parameter distributions, the normal, and Weibull, did not show consistent results for different loading rates.

5. The loading rate affected the value of the fifth percentile in MOR. With an increase in the loading rate from $1 \mathrm{~mm} / \mathrm{min}$ to $10 \mathrm{~mm} / \mathrm{min}$, the empirical $5^{\text {th }}$ percentile value increased by $8.1 \%$, and the theoretical $5^{\text {th }}$ percentile value by $7.2 \%$ and $8.3 \%$ for GEV and $\mathrm{W} 3$, respectively. These differences among the $5^{\text {th }}$ percentile values at different loading rates were noticeably higher than the relative differences between the empirical and theoretical $5^{\text {th }}$ percentile values for the same loading rate. This leads to the conclusion that for $\mathrm{MOR}_{0.05}$ the influence of the loading rate is greater than the influence of the choice of distribution (whether it is an empirical or theoretical distribution).

\section{ACKNOWLEDGMENTS}

The authors are grateful for the support of the Ministry of Education, Science and Technological Development of the Republic of Serbia, Agreement for Funding Scientific Research in 2021 (451-03-9/2021-14/200169).

\section{REFERENCES CITED}

Bohannan, B. (1966). Effect of Size on Bending Strength of Wood Members (Res. Pap. FPL-569), U.S. Department of Agriculture Forest Products Laboratory, Madison, WI, USA.

Butler, M. A., Dahlen, J., Antony, F., Kane, M., Eberhardt, T. L., Jin, H., Love-Myers, K., and McTague, J. P. (2016). "Relationships between loblolly pine small clear specimens and dimension lumber tested in static bending," Wood Fiber Sci. 48(2), 81-95.

Büyüksarı, Ü. (2017a). "Effect of loading rate on mechanical properties of micro-sized oak wood," Maderas-Cienc. Tecnol. 19(2), 163-172. DOI: 10.4067/S0718221X2017005000014 
Büyüksarı, Ü. (2017b). "Effect of loading rate on mechanical properties of micro-size Scots pine wood," BioResources 12(2), 2721-2730. DOI: 10.15376/biores.12.2.27212730

Cunha, C., Tenório, M., Lima, D. F., Rebouças, A., Neves, L. A. C., and Branco, J. M. (2021). "Mechanical characterization of Iroko wood using small specimens," Buildings 11(3), Article number 116. DOI: 10.3390/buildings11030116

D’Agostino, R. B., and Stephens M. A. (1986). Goodness-of-Fit Techniques, Marcel Dekker, New York, NY, USA.

Evans, J. W., Johnson, R. A., and Green, D. W. (2019). Forest Products Laboratory Contributions to the Use of Weibull Distribution in Wood Engineering (Gen. Tech. Rep. FPL-GTR-271), U.S Department of Agriculture Forest Product Laboratory, Madison, WI, USA. DOI: 10.2737/FPL-GTR-271

Gerhards, C. C. (1977). Effect of Duration and Rate of Loading on Strength of Wood and Wood-based Materials (Res. Pap. FPL-RP-283), U.S. Department of Agriculture Forest Product Laboratory, Madison, WI, USA.

Gupta, R., Gebremedhin, K. G., and Grigoriu, M. G. (1992). "Characterizing the strength of wood truss joints," Transactions of the ASAE 35(4), 1286-1290. DOI: $10.13031 / 2013.28731$

Hosking, J. R. M. (1990). " $L$-moments: Analysis and estimation of distributions using linear combinations of order statistics," J. R. Stat. Soc. Series B Stat. Methodol. 52(1), 105-124. DOI: 10.1111/j.2517-6161.1990.tb01775.x

Krajnc, L., Farrelly, N., and Harte, A. M. (2019). "Relationships between wood properties of small clear specimens and structural-sized boards in three softwood species," Holzforschung 73(11), 987-996. DOI: 10.1515/hf-2019-0039

Liska, J. A. (1950). Effect of Rapid Loading on the Compressive and Flexural Strength of Wood (Report No. R1767), U.S. Department of Agriculture Forest Product Laboratory, Madison, WI, USA.

Mandić, M., Porankiewicz, B., and Danon, G. (2015). “An attempt at modelling of cutting forces in oak peripheral milling," BioResources 10(3), 5489-5502. DOI:10.15376/biores.10.3.5489-5502

McNatt, J. D. (1975). Effect of Rate of Loading and Duration of Load on Properties of Particleboard (FPL 270), U.S. Department of Agriculture Forest Product Laboratory, Madison, WI, USA.

Nowak, T., Patalas, F., and Karolak, A. (2021). "Estimation mechanical properties of wood in existing structures - Selected aspects," Materials 14(8), article no. 1941. DOI: $10.3390 / \mathrm{ma} 14081941$

Owens, F. C., Verrill, S. P., Shmulsky, R., and Kretschmann, D. E. (2018). "Distributions of MOE and MOR in a full lumber population," Wood Fiber Sci. 50(3), 265-279. DOI: $10.22382 / \mathrm{wfs}-2018-027$

Porankiewicz, B., Wieczorek, D., Djurkovic, M., Idzikowski, I., and Węgrzyne, Z. (2021). "Modelling cutting forces using the moduli of elasticity in oak peripheral milling," BioResources 16(1), 1424-1437. DOI: 10.15376/biores.16.1.1424-1437

Rao, A. R., and Hamed, K. H. (2000). Flood Frequency Analysis, CRC Press, Boca Raton, FL, USA. DOI: 10.1201/9780429128813

Sankarasubramanian, A., and Srinavasan, K. (1999). "Investigation and comparison of sampling properties of $L$-moments and conventional moments," J. Hydrol. 218, 1334. DOI: 10.1016/S0022-1694(99)00018-9 
SRPS EN 384 (2019). "Structural timber - Determination of characteristic values of mechanical properties and density," Institute for Standardization of Serbia, Belgrade, Serbia.

SRPS EN 408 (2014). "Timber structures — Structural timber and glued laminated timber - Determination of some physical and mechanical properties," Institute for Standardization of Serbia, Belgrade, Serbia.

SRPS ISO 13061-1 (2015). "Physical and mechanical properties of wood - Test methods for small clear wood specimens - Part 1: Determination of moisture content for physical and mechanical tests," Institute for Standardization of Serbia, Belgrade, Serbia.

SRPS ISO 13061-2 (2015). "Physical and mechanical properties of wood - Test methods for small clear wood specimens - Part 2: Determination of density for physical and mechanical tests," Institute for Standardization of Serbia, Belgrade, Serbia.

SRPS ISO 13061-3 (2015). "Physical and mechanical properties of wood - Test methods for small clear wood specimens - Part 3: Determination of ultimate strength in static bending," Institute for Standardization of Serbia, Belgrade, Serbia.

SRPS ISO 13061-4 (2015). "Physical and mechanical properties of wood - Test methods for small clear wood specimens - Part 4: Determination of modulus of elasticity in static bending," Institute for Standardization of Serbia, Belgrade, Serbia.

Stedinger, J. R. (1980). "Fitting log normal distributions," Water Resour. Res. 16(3), 481490. DOI: 10.1029/WR016i003p00481

Tamrakar, S., and Lopez-Anido, R. A. (2010). "Effect of strain rate on flexural properties of wood plastic composite sheet pile," Forest Prod. J. 60(5), 465-472. DOI: 10.13073/0015-7473-60.5.465

Vogel, R. M., and Fennessey, N. M. (1993). " $L$ moment diagrams should replace product moment diagrams," Water Resour. Res. 29(6), 1745-1752. DOI: 10.1029/93WR00341

Vogel, R. M., and Wilson, I. (1996). "Probability distribution of annual maxixum, mean, and minimum streamflows in the United States," J. Hydrol. Eng. 1(2), 69-76. DOI: 10.1029/93WR00341

Article submitted: October 25, 2021; Peer review completed: January 1, 2022; Revised version received and accepted: January 23, 2022; Published: January 26, 2022.

DOI: 10.15376/biores.17.1.1818-1835 